\title{
Clinical Trial Data as Public Goods: Fair Trade and the Virtual Knowledge Bank as a Solution to the Free Rider Problem - A Framework for the Promotion of Innovation by Facilitation of Clinical Trial Data Sharing among Biopharmaceutical Companies in the Era of Omics and Big Data
}

\author{
Nikolaos Evangelatos ${ }^{a, b}$ Matthias Reumann ${ }^{a, c}$ Hans Lehrach $^{\text {d, e }}$ Angela Brand $^{a}$ \\ a UNU-MERIT, Faculty of Humanities and Sciences, Maastricht University, Maastricht, The Netherlands; b University Clinic \\ for Emergency and Intensive Care Medicine, Paracelsus Medical University (PMU), Nuremberg, Germany; \\ ${ }^{C}$ IBM Research - Zurich, Rüschlikon, Switzerland; ${ }^{\mathrm{d}}$ Max Planck Institute for Molecular Genetics, and ${ }^{\mathrm{D}}$ Dahlem Centre for \\ Genome Research and Medical Systems Biology, Berlin, Germany
}

\section{Key Words}

Big Data $\cdot$ Clinical trial $\cdot$ Personalized medicine $\cdot$ Data sharing · Free rider · Innovation · Public goods · Virtual Knowledge Bank

\begin{abstract}
Background: Knowledge in the era of Omics and Big Data has been increasingly conceptualized as a public good. Sharing of de-identified patient data has been advocated as a means to increase confidence and public trust in the results of clinical trials. On the other hand, research has shown that the current research and development model of the biopharmaceutical industry has reached its innovation capacity. In response to that, the biopharmaceutical industry has adopted open innovation practices, with sharing of clinical trial data being among the most interesting ones. However,
\end{abstract}

due to the free rider problem, clinical trial data sharing among biopharmaceutical companies could undermine their innovativeness. Method: Based on the theory of public goods, we have developed a commons arrangement and devised a model, which enables secure and fair clinical trial data sharing over a Virtual Knowledge Bank based on a web platform. Our model uses data as a virtual currency and treats knowledge as a club good. Results: Fair sharing of clinical trial data over the Virtual Knowledge Bank has positive effects on the innovation capacity of the biopharmaceutical industry without compromising the intellectual rights, proprietary interests and competitiveness of the latter. Conclusion: The Virtual Knowledge Bank is a sustainable and selfexpanding model for secure and fair clinical trial data sharing that allows for sharing of clinical trial data, while at the same time it increases the innovation capacity of the biopharmaceutical industry.

(c) 2016 S. Karger AG, Basel

\section{KARGER}

E-Mail karger@karger.com

www.karger.com/phg
(C) 2016 S. Karger AG, Basel

$1662-4246 / 16 / 0194-0211 \$ 39.50 / 0$
Dr. Nikolaos Evangelatos

University Clinic for Emergency and Intensive Care Medicine Paracelsus Medical University (PMU)

Professor Ernst-Nathan-Str. 1, DE-90419 Nuremberg (Germany)

E-Mail evangelatos@merit.unu.edu 


\section{Introduction}

Relevant research has shown that the rate of new drugs production has remained relatively constant in the last decades since 1950, with biotechnology not delivering on its promise to increase the rate of innovation in the biopharmaceutical industrial sector. Taking into account the unprecedented investment in biopharmaceutical research and development $(\mathrm{R} \& \mathrm{D})$ during the same period [1], this may suggest that the current R\&D model of the biopharmaceutical industry has reached its innovation capacity [2].

On the other hand, the elucidation of our genomic background has allowed for the recognition of causative pathways leading to disease and for the development of novel agents, designed and developed according to certain genomic profiles. Claiming a different epistemological status concerning the rationality of their development, the new agents essentially change the blockbuster business model to a new one, where targeted therapies have a better efficacy but smaller markets and shorter product life cycles. Coupled with changes in the global economic landscape, which impose radical reforms in the reimbursement of pharmaceuticals, the new era of personalized medicine poses great challenges to the biopharmaceutical industry [3].

In response to those challenges, the biopharmaceutical industry has been trying to redesign the current $\mathrm{R} \& \mathrm{D}$ model in order to promote and accelerate drug development. Recognizing the key role of the clinical trials and the need to reduce high attrition rates and increasing costs, the biopharmaceutical industry has moved from the classical clinical development approach of the many, sequential, distinct phases, to a more integrative approach with adaptive clinical trial designs [4].

A complementary response to the new challenges regards the adoption of new business models in the drug development process. Although differences between industrial sectors pose difficulties, developments in the computer industry have rendered open innovation practices appealing to the field of the biopharmaceutical industry as well [5]. Indeed, despite major differences when compared to the successful open source software movement (such as different research dynamics, different economic and time scales, lack of clinical trial equivalents, lighter regulation, different intellectual property landscapes, to name but a few) [6], the biopharmaceutical industry has started adopting open innovation practices [7].
Although the concept of open innovation has different interpretations among corporate organizations, it essentially refers to 'the use of purposive inflows and outflows of knowledge to accelerate internal innovation, and expand the markets for external use of innovation, respectively' [8]. Among the most interesting open innovation practices in the biopharmaceutical sector is the sharing of data from clinical trials. Apart from enabling secondary analyses, which allow for increased safety surveillance and improved public confidence in clinical trial results, sharing of clinical trial data also facilitates the exploration of unrecognized scientific questions, thus leading to informed strategic decisions regarding allocation of resources by the biopharmaceutical companies. Potential beneficiaries are practically all stakeholders along the health care value chain [9]. Despite its many potential benefits, the sharing of clinical trial data has also raised concerns regarding, among others, the impacts of re-analyses, use of data for commercial gain and patient and investigator confidentiality compromise [10]. In order to address these concerns, criteria for the conduction of re-analyses have already been proposed [11].

Recognizing the potential of clinical trial data sharing, governmental agencies, scientific journals, and private initiatives have been working towards an effective datasharing culture, facilitating access to de-identified patient-level data from both publicly funded and industrysponsored clinical trials [12-14]. A recent evaluation has shown that the academic community has enthusiastically welcomed these initiatives [15].

Within the strong intellectual property rights environment of the biopharmaceutical industrial sector, sharing of clinical trial data has mainly taken the form of Public Private Partnerships (PPP) between publicly funded research institutes and private biopharmaceutical companies [16]. Research has highlighted both the advantages and disadvantages of PPP as compared to traditional, vertically integrated, internal R\&D department-to-market approaches $[17,18]$.

However, in reality PPP is not a new idea. Research has revealed the existence of common academic-industry projects and commissioned research, predominantly in the field of microbiology and biochemistry, already in the interwar period. This research provides evidence for the existence of cooperation between the pharmaceutical industry and academic science long before the era of modern biotechnology [19].

Open innovation practices, in the form of clinical trial data sharing, are also supported by the European Fed- 
eration of Pharmaceutical Industries and Associations (EFPIA) [20]. Initiatives such as EFPIA's Clinical Trial Data Portal Gateway [21] and ClinicalStudyDataRequests.com [22] provide access to anonymized patientlevel data from industry-sponsored clinical trials. Researchers can submit enquiries to access data from clinical trials. The relevant proposals are reviewed by an Independent Review Panel without participation of the study sponsor. In case of approval, and with permission from the study sponsor, researchers sign a Data Sharing Agreement and get access to data on a password-protected website. In this case, clinical trial data is being shared one way, from a biopharmaceutical company to a researcher.

However, radically advanced open innovation business models, in the form of Private Private Partnerships (PrPrP) between biopharmaceutical companies, which could revolutionize the clinical trial data sharing landscape, are still missing.

\section{Knowledge as Public Good and the Free Rider Problem}

The main reason for the reluctancy of private companies to share their $\mathrm{R} \& \mathrm{D}$ data (in the form of clinical trial data) with other companies and benefit from the sharing relies on their concerns regarding compromise of their intellectual rights, proprietary interests, and competitiveness. Sharing of clinical trial data could undermine patentability and, therefore, adversely affect a company's interests, whereas implementation of clinical trial results disclosure policies could lead to premature submission of marketing license applications without enough safety and efficacy data. The conflict between the long-term common interest and the short-term individual one is being resolved in favor of the latter. The problem can be summarized as the risk of free riding on the efforts of others. In his seminal 1968 paper, G. Hardin [23] described the tragedy of the commons referring to the free riding problem. Individuals, acting independently and rationally according to each one's self-interest, behave contrary to the whole group's long-term best interests by depleting some common resources. In this sense, firms are reluctant to provide open access to their data as this would render them vulnerable to competitors' free riding on their R\&D investments.

On the other hand, research in the era of Omics (genomics, epigenomics, proteomics, metabolomics, exposomics, etc.) is generating vast amounts of diverged data in electronic format. In the biopharmaceutical industry,

Fair Clinical Trial Data Sharing and the Virtual Knowledge Bank meaningfully aggregated and interpreted clinical trial data generates information. Accommodated in a highly complex environment, consisting of a certain perceived context and practical experience, information has the potential to be transformed to socially meaningful scientific knowledge, which can in turn lead to innovation [24]. However, management, analysis and effective exploitation of data require expertise input from different sectors, readiness for cooperation and willingness to share information with different organizations. Knowledge in the above context has been increasingly conceptualized as a public good [25].

\section{Knowledge and Common-Pool Natural Resources}

Considering knowledge as a public good and based on the analogies between the cultural and the natural environment [26], researchers have highlighted the similarities between governance of knowledge, as a public good, and governance of common-pool natural resources [27].

In order to overcome the free rider problem in the case of common-pool resources, diverged economic and political solutions have been advocated and several institutions have been developed. As far as common-pool natural resources are concerned, governing approaches range from adopting a central regulation (i.e. ownership and regulation by the state) to espousing a private corporate system (i.e. ownership by for-profit companies). However, as already mentioned, both systems have shown an unsatisfactory performance in addressing the problem of lacking innovativeness.

An alternative would be, as Elinor Ostrom showed by studying the common-pool natural resources, the adoption, by the members of the community, of a selfregulation model relying mainly on a moral code. Within the framework of the Institutional Analysis and Development (IAD) approach [28], several commons arrangements, that overcome the problem of overexploitation in sharing common-pool natural resources, have been studied. The study of both successful and unsuccessful commons arrangements has largely identified their underlying design principles which rest on horizontal, non-hierarchical self-regulation models based on the implementation of moral codes of behavior [29].

The similarities to common-pool natural resources [30], together with the enthusiasm about knowledge as a shared resource in the sense of a public good, have led to uncritical embracement of commons arrangements 
for knowledge resources, without, however, the deeper understanding of their benefits and risks, which is necessary for policy making that actually promotes innovation.

\section{Knowledge as a Club Good}

Indeed, despite the profound similarities between knowledge and natural resources, there are also important differences. Unlike other assets, knowledge, particularly in its digital form, can be replicated and distributed at practically no cost. At the same time, the original source (as well as the copy) remains available for others to use. From the theory of public goods point of view, knowledge is, in this sense, a non-rivalrous good.

On the other hand, sharing of data and, therefore, conceptualization of knowledge as a shared resource, requires not only that information is freely accessible, but also that potential recipients are able to devote time and resources in order to assimilate it as knowledge [31]. This process depends largely on the absorptive capacity of the firms and their ability to turn information to knowledge and finally into innovation, which, in the case of the biopharmaceutical industry, would mean accelerated drug development [32]. This suggests that unregulated, publicly available access to patient-level data from industry clinical trials should not be expected to promote innovativeness. Indeed, in order to produce meaningful results, sharing of knowledge should ideally take place among entities that have the capability to transform information to knowledge and hence innovation.

Since transformation of information to knowledge requires certain capabilities, excludability criteria (when it comes to sharing of knowledge) are already present. In this sense, knowledge as a non-rivalrous but excludable good (even if freely accessible) is not a common-pool resource, but rather a club good.

Contrary to that, common-pool natural resources are rivalrous but non-excludable [33]. The principles that govern natural resources commons are purposed to settle concerns related to rivalry; excludability criteria do not apply, at least theoretically. Moreover, and perhaps most importantly, the biopharmaceutical industrial sector consists of for-profit organizations. As profit per se is not value laden, moral codes that govern the behavior of the participants of a commons are not applicable in the case of a knowledge commons consisting of biopharmaceutical for-profit companies.

\section{Fair Trade as a Solution to the Free Rider Problem: The Virtual Knowledge Bank}

In this context, overcoming the free rider problem in the case of knowledge commons should rely on more appropriate solutions that combine economic rationale with political willingness.

Clinical trial data in electronic format can be considered information resources. Developments in the field of information and communication technology (mainly the Internet) have reduced the transaction costs for pooling and sharing information resources to a minimum. In this setting, potential participants of a knowledge commons are able to contact each other and share their knowledge by executing transactions between them without transaction costs. On the other hand, as mentioned elsewhere, excludability criteria are, in practice, applied in the sharing of knowledge even if information is freely accessible. In case the costs of the exclusion mechanisms do not exceed the benefit from the participation in a knowledge commons, club goods (i.e. non-rivalrous but excludable) can emerge spontaneously [34]. Taking into account the individuals' self-interested willingness to pay, the main challenge is to devise the appropriate practical arrangements which allow one party to benefit without causing more harm (in absolute terms of value) to the other contracting party [35].

Considering knowledge a club good and based on the above, we provide a suggested framework for overcoming the free rider problem in the case of the biopharmaceutical industry, thus promoting data sharing among biopharmaceutical for-profit companies. We propose the construction of a web-based platform for the exchange of data (genomics, epigenomics, proteomics, metabolomics, exposomics, etc.) from clinical trials. In our model, the platform works as a Virtual Knowledge Bank (VKB), where clinical trial data in electronic format is stored as information resources. Depositors are private for-profit biopharmaceutical companies entitled to deposit and also withdraw information resources (i.e. data from clinical trials) from the VKB. Transactions will be regulated by applying only one (excludability) criterion: companies will be allowed to download the same amount of data, quantitatively and qualitatively, which they upload. In essence, information resources, in the form of clinical trial data, are being used as a currency for carrying out transactions. By treating knowledge as a club good, our model facilitates knowledge transfer without an impact on the intellectual property rights of the involved companies. Indeed, the latter do not patent knowledge in the form of 


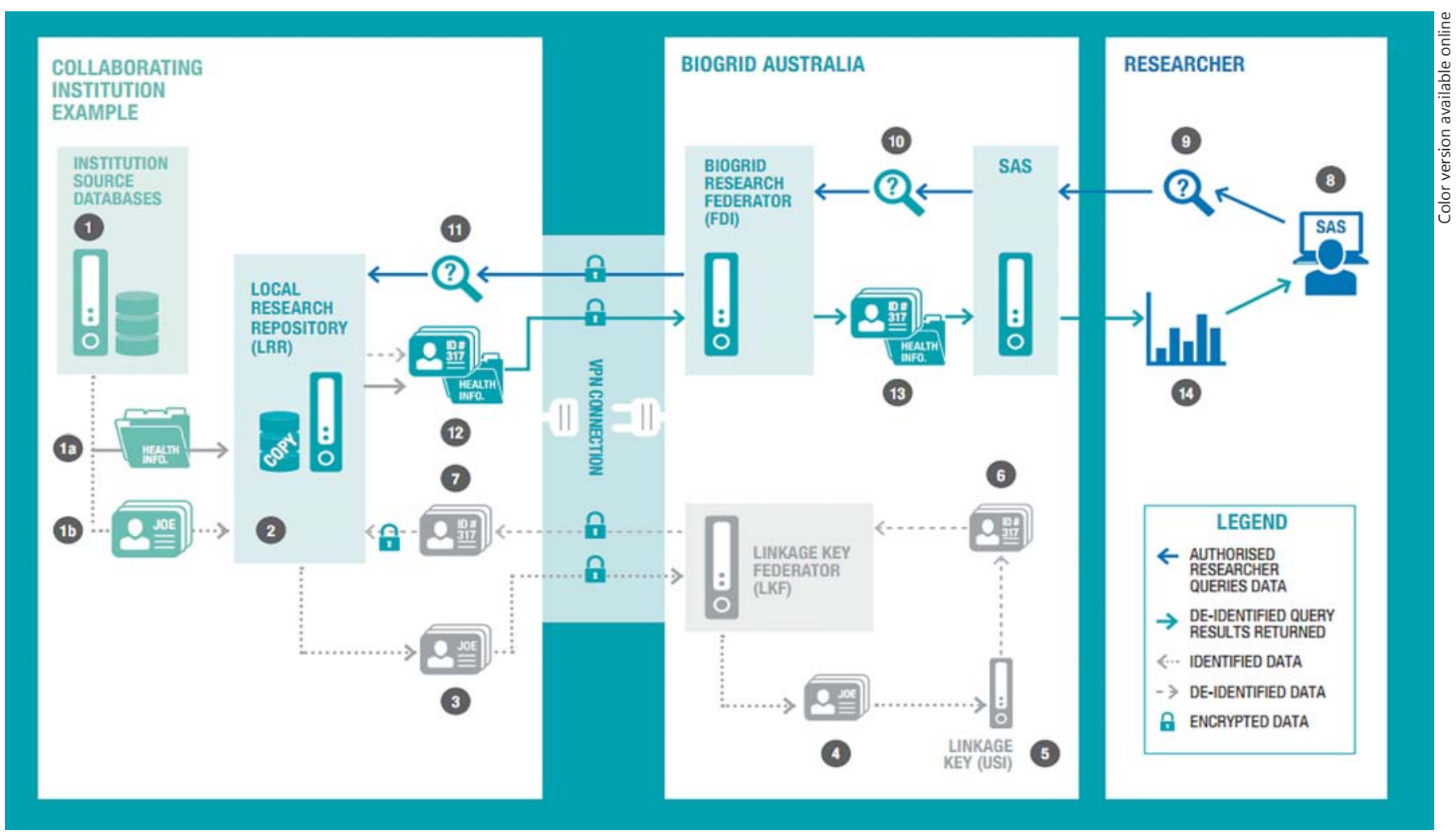

Fig. 1. Architectural design of BioGrid. Used with the permission of BioGrid Australia Ltd.

clinical trial data but rather applied knowledge in the form of medicinal formulas, etc. This way, the landscape changes without compromising the competitiveness of the involved parties.

\section{Technical Implementation for Data Sharing}

The technological implementation of the VKB can be manifold and has to address a plethora of issues ranging from what kind of data are being shared to where data is stored, who gets and controls access, and how data transfer is managed. Here, we will only describe the BioGrid Australia platform [36] that has been designed for sharing of research data as one solution to be transferred to the VKB setting. BioGrid Australia operates as a federated data-sharing platform for translational research in medicine and biology. Data is linked in a secure infrastructure that provides privacy protection and is ethically approved. The data is distributed in the sense that each member and collaborator stores and retains control of their data including granting access. The BioGrid Australia platform de-identifies and links data sources that are delivered to authorized researchers. Authorization is given by the "key holder', a person nominated by the owning party of the respective data. Each member can request access to data that is described in a catalogue containing meta-data. While the request and data exchange between two parties are managed via the BioGrid platform, it remains an exchange between the two research organizations. In this sense, BioGrid Australia acts as research platform and data gateway. Its high level architectural design is illustrated in figure 1.

Similarly, this kind of infrastructure can be implemented in a cloud environment where the data can be moved into the same cloud, while control and access are still maintained by the owning institution. A further level of data management would have to be introduced, which deals with the accounting for data transfer core element of the VKB. Currently, the BioGrid Australia model allows any member to receive any amount and kind of data within the network based on permission of the owning party. It does not include a mechanism to manage depositing data in return for getting access to already stored data. 


\section{Creating a Dynamic Ecosystem}

To create a dynamic ecosystem around our proposed approach, there are plenty of challenges that need to be addressed. Future work that will engage the scientific community through initiatives such as the European Alliance for Personalised Medicine (EAPM) will discuss and create solutions. Here we outline some of the major challenges.

The simplest interaction in the VKB model is that each contributing party (i.e. a biopharmaceutical company) has deposited (in the sense that it has made it potentially available to other contributing parties) its data from a number of clinical trials. If a third party would like to use any of the VKB data, then it should deposit (i.e. also make potentially available to other parties who are willing to 'pay' for it) the same amount, quantitatively and qualitatively, of data that it requires. For example, 1 terabyte of genomic data from a phase II clinical trial requested needs to be matched with 1 terabyte of data from another phase II clinical trial. The same happens when a third party needs access to data (e.g. proteomic data) from a phase III clinical trial. This set of data is more 'expensive' in the sense that one has to provide access to own data, also from a phase III clinical trial, in order to be able to get access to the requested set of data. In this case, 'uploading' (in the sense of making potentially available to other interested parties) data from a clinical trial phase II would not be enough. It is important to highlight the fact that the data is made potentially available to every party which may be interested in it. It is not a peer-to-peer transaction between two parties, but rather a unique arrangement, where every member of the club can make use of the club goods to the extent that it itself has contributed (and continues to contribute) to the construction of the club goods by providing its own data. If a party needs access to another set of data, then this party will be required to deposit (make potentially available) another set of its own data in order for the transaction to be conducted. Thus, with every transaction, the VKB grows richer by the amount of data used in the transaction. That leads essentially to a self-expanding Biobank that grows more attractive with every transaction conducted. Consequently, it is clear that the VKB will attract more and more clients (biopharmaceutical companies), as the deposited data will provide possible answers to many parties. These parties will in turn be willing to deposit some of their own data from clinical trials, in order to gain access to data already deposited in the bank, which may be useful for their research and commercial activities.
However, not all stakeholders who want to benefit from such a Biobank might have the same amount of data or the data they need to get access to a larger data set. Hypothetically speaking, a small biotechnology start-up company or a research group without commercial interest has already deposited a certain amount of data but requires twice as much data or a tenfold of data to continue their research and development. The system should not deny this company access to the needed data but rather allow negotiation of contribution in a different kind that fosters the community.

In this sense, we have two extreme cases and many shades in between that need to be addressed:

1 A stakeholder produces large amounts of data and does not need data from other sources: how can this stakeholder be incentivized to deposit all her/his data into the Biobank so that the community can benefit from it?

2 A stakeholder does not have any data but wants to build services and products using the data or through analysis of the data for product and service development: how can this stakeholder be engaged to 'pay back' the amount of data that it gets access to?

Furthermore, since data is becoming the suggested currency in our model, we need to address the issue of different data and its different value. Data is heterogeneous and a large variety of data exists. A certain data type can be more valuable to resolve one challenge but worth nothing for another challenge. Who determines the value of data? Is depositing a whole genome sequence worth more than two exome sequences? Do base pairs count as common denominator? Comparing genomic data with proteomic data, health records or data from social media further illustrates the complexity of data as currency. It becomes apparent that the analogy of currencies is very close to comparing the value of different data types. While monetary currency conversion is established throughout the world economies, the value of one data type versus another is yet not clear. Even data of the same type comes in many shades: a well-managed and curated data set (that also extends longitudinally) should be more valuable than a data set that contains noise and errors, like a diamond's size, purity, shape and color determine its value. In this context, data harmonization and the ability to record linkage (in the sense that one has to be able to combine data sets to create synergies) play an important role. It has not escaped our notice that the function of the VKB resembles to a certain extent the function of a stock market where investors are required to adjust their 'portfolio' according to the trends and expectations, as far as its 
Table 1. How VKB promotes innovation

Enables regulated sharing of knowledge between stakeholders

Every transaction increases the possibility that companies find answers to their questions

Small biotechnology companies gain access to data from other research fields

Decreases attrition rates

Lowers financial barriers

Past R\&D efforts find new uses - decreased R\&D costs

Enables detection of potential synergies - leads to future cooperations

Highlights use of data as virtual currency

druggability is concerned, related to a certain set of data from a clinical trial.

The VKB also needs to provide data license agreements. The contributing stakeholders will want their data to be used in a certain way and they need to have the guarantee of freedom of action on data that they access within the terms of a data license agreement. Again, an analogy can be drawn to the open source software community. Open source software licenses come in many shades and forms, such as the GNU, GPL or Apache, to name but a few. Similar licenses have to be defined for data use from the VKB.

One of the most important aspects to consider is the right of the participating patients to their own data. In a sense, data generated in a trial should perhaps not only be a property of the researcher/company, but rather coowned by the participating patients who generated it. Initiatives such as MIDATA.coop enable citizens to control access to their personal data, possibly allowing groups of participants to have a voice in data sharing among biopharmaceutical companies as well $[37,38]$.

Security concerns will always arise but can be addressed as exemplified by the BioGrid Australia platform where security and encryption is a core part of the implementation. Bitcoin and block chain offer new opportunities for data security so that data can be traced and tracked $[39,40]$.

\section{Benefits for Innovation}

Recognizing the significance of clinical trial data sharing, the International Committee of Medical Journal Editors (ICMJE) has recently announced its intention to require from authors, as a condition for publication of clinical trials in the member journals, to share the de-identified individual patient data (IPD) of the study within 6 months after publication. The requirement refers not only to publicly funded clinical trials but also to clinical trials sponsored by the industry [41]. In our view, although the ini- tiative of the ICMJE will result in increased confidence and public trust in the results of clinical trials, it does not sufficiently take into account the impact of clinical trial data sharing on the innovation capacity of the biopharmaceutical companies.

Initiatives such as the EAPM firmly believe that sharing of clinical trial data can be effectively implemented by taking into consideration its impact on all stakeholders along the health care chain value. In our era, public interest is increasingly being taken into account, with conventional capitalistic business models, characterized by a strong intellectual property rights environment, gradually making space for open innovation practices and commons arrangements. Our model of data sharing is compatible with the moral obligation to treat clinical trial data as public goods without, however, compromising the intellectual property rights of the involved biopharmaceutical companies. Furthermore, with excludability relying on a fair and affordable criterion, our model introduces fair trade in the field of clinical trial data sharing. Apart from responding to the ethical obligation towards the public good, our model fosters innovation in many ways. Indeed, regulated sharing of clinical trial data enhances the innovation capacity of the biopharmaceutical companies and promotes the innovativeness of the sector as a whole (table 1). First, every transaction increases the property of the bank, thus rendering it more attractive to potential transactors, who have more possibilities to find the answers to their problems at a cost they can afford. Second, especially small biotechnology companies, the majority of which conduct basic research or clinical trials up to phase II, are expected to profit by engaging in transactions over the virtual bank. Indeed, big pharmaceutical firms are in position to test an alternative hypothesis in-house and at affordable cost, whereas shared data can be proven extremely useful to small biotech companies, which, due to limited resources and lack of relevant expertise, cannot set up and conduct phase III clinical trials or reorientate their research efforts in order to test alternative hypotheses. Moreover, as small 
biotechnology companies are often startups or spin-offs with limited resources, they are dedicated to only one research field. The VKB enables them to gain data from other research fields, recognize synergies, and expand their overview of the research and business arena. Third, large biopharmaceutical companies also benefit since they can acquire data from competitors working on similar areas, a fact that will allow them to reorientate their research efforts accordingly, thus reducing the high attrition rates of the drug development process. Fourth, being compatible with the premises of the new emerging business models and commons arrangements, the unique characteristic of using data as a virtual currency liberates companies from the restrictions imposed by their economic status and financial obligations, thus lowering the relevant economic barriers. Fifth, R\&D efforts of the past, which did not find their way to the market as a new medicine or diagnostic device, can find new uses as a currency used to acquire data, which are of use for current R\&D efforts. This way, $R \& D$ costs for the development of a new medicine or devise are in effect being reduced, as the relevant data may be re-used as a currency for future transactions and for the acquisition of data used for new R\&D efforts. Last but not least, knowledge of data from participants highlights potential synergies and has the potential to lead to future cooperations.

\section{Conclusion}

Knowledge in the era of Omics and Big Data has been increasingly conceptualized as a public good, with governmental agencies, scientific journals and private initia- tives working towards an effective data-sharing culture. On the other hand, research has shown that the current R\&D model of the biopharmaceutical industry has reached its innovation capacity. In response to that, the biopharmaceutical industry has adopted clinical trial data sharing as a means to promote innovation. However, due to the free rider problem, freely accessible clinical trial data could de-incentivize the biopharmaceutical industry, thus having a negative impact on the development of new medicines. We have proposed a commons arrangement and devised a model, which enables secure and fair clinical trial data sharing over a VBK based on a web platform. Using data as a virtual currency and treating knowledge as a club good, our model of data sharing is compatible with the moral obligation to share clinical trial data without compromising the competitiveness of the involved biopharmaceutical companies. The VBK is a sustainable and self-expanding framework for secure and fair clinical trial data sharing that increases the innovation capacity of the biopharmaceutical industry. Convinced of the potential of the VKB to revolutionize clinical trial data sharing among biopharmaceutical companies, our group in the EAPM is currently working, in cooperation with representatives of the biopharmaceutical industry, to resolve the issues mentioned above and to develop the web-based platform.

\section{Disclosure Statement}

The authors have no conflicts of interest to disclose.

\section{References}

1 DiMasi JA, Grabowski HG: The cost of biopharmaceutical R\&D: is biotech different? MDE Manage Decis Econ 2007;28:469-479.

2 Munos B: Lessons from 60 years of pharmaceutical innovation. Nat Rev Drug Discov 2009;8:959-968.

3 Vogler S, Zimmermann N, Leopold C, Joncheere KD: Pharmaceutical policies in European countries in response to the global financial crisis. South Med Rev 2011;4:69-79.

$\checkmark 4$ Orloff J, Douglas F, Pinheiro J, Levinson S, Branson M, Chaturvedi P, Ette E, Gallo P, Hirsch G, Mehta C, Patel N, Sabir S, Springs S, Stanski D, Evers MR, Fleming E, Singh N, Tramontin T, Golub H: The future of drug development: advancing clinical trial design. Nat Rev Drug Discov 2009;8:949-957.
-5 Chesbrough H: An audience with... Henry Chesbrough. Interview by Asher Mullard. Nat Rev Drug Discov 2013;12:338-339.

6 An open-source shot in the arm? The Economist, June 12, 2004.

7 Munos B: Can open-source R\&D reinvigorate drug research? Nat Rev Drug Discov 2006;5: 723-729.

8 Chesbrough HW: Open innovation: the new imperative for creating and profiting from technology. Harvard Business School Press, September 30, 2005.

-9 Mello MM, Francer JK, Wilenzick M, Teden $\mathrm{P}$, Bierer BE, Barnes M: Preparing for responsible sharing of clinical trial data. N Engl J Med 2013;369:1651-1658.
10 Krumholz HM, Peterson ED: Open access to clinical trials data. JAMA 2014;312:1002-1003.

11 Christakis DA, Zimmerman FJ: Rethinking reanalysis. JAMA 2013;310:2499-2500.

12 Godlee F, Groves T: The new BMJ policy on sharing data from drug and device trials. BMJ 2012;345:e7888.

13 http://www.ema.europa.eu/docs/en_GB/document_library/Other/2014/10/WC500174796. pdf (accessed November 8, 2015).

14 Nisen P, Rockhold F: Access to patient-level data from GlaxoSmithKline clinical trials. $\mathrm{N}$ Engl J Med 2013;369:475-478.

15 Strom BL, Buyse M, Hughes J, Knoppers BM: Data sharing, year 1 - access to data from industry-sponsored clinical trials. N Engl J Med 2014;371:2052-2054. 
16 Institute of Medicine: Sharing Clinical Research Data: Workshop Summary. Washington, National Academies Press, 2013.

17 Health and Economic Analysis for an Evaluation of the Public-Private Partnerships in Health Care Delivery across Europe. http:// ec.europa.eu/health/expert_panel/opinions/ docs/003_assessmentstudyppp_en.pdf (accessed November 21, 2015).

$18 \mathrm{http}: / / \mathrm{ppp}$.worldbank.org/public-privatepartnership/overview/ppp-objectives (accessed November 21, 2015).

19 Marks. HM: The Progress of Experiment: Science and Therapeutic Reform in the United States, 1900-1990, ed 1. Cambridge Studies in the History of Medicine, 2000.

20 http://phrma.org/sites/default/files/pdf/ PhRMAPrinciplesForResponsibleClinicalTrialDataSharing.pdf (accessed March 24, 2016).

21 http://transparency.efpia.eu/responsible-data-sharing/efpia-clinical-trial-data-portalgateway (accessed November 16, 2015).

22 https://www.clinicalstudydatarequest.com (accessed November 16, 2015)

23 Hardin G: The tragedy of the commons. The population problem has no technical solution; it requires a fundamental extension in morality. Science 1968;162:1243-1248.
24 Miller B, Malloy MA, Masek E, Wild C: Towards a framework for managing the information environment. Inf Knowl Syst Manage 2001;2:359-384.

25 Archibugi D, Filippetti A: Knowledge as global public good; in Archibugi D, Filippetti A (eds): Handbook of Global Science, Technology and Innovation. Oxford, Wiley-Blackwell, 2015, pp 483-597.

26 Frischmann BM: Cultural Environmentalism and the Wealth of Networks. Univ Chic Law Rev 2007;74:1083.

27 Frischmann BM, Madison MJ, Strandburg KJ: Governing Knowledge Commons. Oxford University Press, 2014.

28 Ostrom E: The institutional analysis and development framework and the commons. Cornell L Rev 2010;95:807-816.

29 Hess C, Ostrom E: Understanding Knowledge as a Commons: From Theory to Practice. MIT Press, 2007.

30 Hess C, Ostrom E: Ideas, artifacts and facilities: information as a common-pool resource. Law Contemp Probl 2003;66:111-145.

31 Gyuris F: Basic education in communist Hungary. A commons approach. Int J Commons 2014;8:531-553.

32 Cohen WM, Levinthal DA: Absorptive capacity: a new perspective on learning and innovation. Adm Sci Q 1990;35:128-152.
33 Ostrom E, Ostrom V: The Meaning of American Federalism: Constituting a Self-Governing Society. San Francisco, Institute for Contemporary Studies, 1991.

34 Buchanan JM: An economic theory of clubs. Economica. New Series 1965;32:1-14.

35 Coase RH: The problem of social cost. J Law Econ 1960;3:1-44.

36 https://www.biogrid.org.au (accessed March $22,2016)$

37 https://www.midata.coop (accessed March 24, 2016).

38 Becnel LB, Pereira S, Drummond JA, Gingras M-C, Covington KR, Kovar CL, Doddapaneni HV, Hu J, Muzny D, McGuire AL, Wheeler DA, Gibbs RA: An open access pilot freely sharing cancer genomic data from participants in Texas. Sci Data 2016;3:160010.

39 Nakamoto S: 2008. Bitcoin: a peer-to-peer electronic cash system. https://bitcoin.org/ bitcoin.pdf (accessed March 22, 2016).

40 Bonneau J, Miller A, Clark J, Narayanan A, Kroll JA, Felten EW: Research perspectives and challenges for bitcoin and cryptocurrencies. Security and Privacy (SP), 2015 IEEE Symposium, pp 104-121.

41 Sharing Clinical Trial Data - A Proposal from the International Committee of Medical Journal Editors. NEJM.org, January 20, 2016. 